\title{
Herzinsuffizienz
}

\section{Medikamente und Medizintechnik}

Die Herz-Kreislauf-Medizin kann wie kaum eine andere medizinische Disziplin auf eine große Erfolgsgeschichte in den letzten Jahrzehnten zurückblicken. Die altersadjustierte Inzidenz schwerwiegender Herz-Kreislauf-Erkrankungen und insbesondere die damit verbundene hohe Morbidität und Mortalität konnten teilweise deutlich reduziert werden. Die Anzahl an Herzinfarkten nimmt ab, ebenso gesenkt werden konnte die Sterblichkeit dieser Erkrankung.

Bedingt sind diese Fortschritte durch bessere Präventionsmaßnahmen, besseres Risikofaktormanagement und eine optimierte Behandlung der Patienten im Akutfall. Das bessere Management, z. B. des Herzinfarktes, und die allbekannte demografische Entwicklung führen jedoch dazu, dass die Herzinsuffizienz nichts von ihrer Allgegenwart verloren hat - ganz im Gegenteil. Die Prävalenz dieser Erkrankung ist eher steigend, und die Komplikationsrate inklusive Tod bleibt trotz beachtlicher Erfolge hoch. Nicht zuletzt deshalb handelt es sich daher um einen der wichtigsten Kostentreiber in unserem Gesundheitssystem.

Gelegenheit genug also, um nochmals die wesentlichen Bausteine der Herzinsuffizienztherapie miteinander zu diskutieren. Die Grundlage ist die medikamentöse Behandlung. Hier wurden erstaunliche Fortschritte in den letzten Jahren erzielt, die Schritte sind gegenwärtig jedoch kleiner geworden. Welche weiteren Perspektiven und Neuentwicklungen haben wir zu erwarten, welche neuen Entwicklungen sind für unsere Praxis relevant? Der zweite, immer wichtiger gewordene Baustein ist die sogenannte Devicetherapie mit Defibrillatoren und Resynchronisationstherapien. Anlässlich neuer Studien ist es zu einer weiteren Verfeinerung der Therapie gekommen. Welche Dinge hiervon sind tatsächlich relevant für unsere Patienten, welche sind nur dazu angetan, die Interessen der Industrie zu fördern?

Versagen diese Bausteine, steht am Ende oft die Herztransplantation oder das künstliche Herz. Auch hier sind insbesondere in Bezug auf das künstliche Herz neue Fortschritte zu verkünden.

Schließlich werden diese therapeutischen Maßnahmen ergänzt um weitere Behandlungsmodalitäten, z. B. im Bereich der Mitralklappe. Dieses Kapitel gehört zu den aufregendsten gegenwärtigen Themen in der Herzmedizin, von der Operation bis hin zu der atemberaubenden Neuentwicklung neuer Interventionstechniken jetzt auch im Bereich der Mitralklappe.

Wir hoffen sehr, dass wir Ihnen ein interessantes Konzeptheft zusammenstellen konnten und wünschen Ihnen viel Freude und Genuss bei der Lektüre.

Ihr

Prof. Dr. G. Nickenig 